\title{
A Spatially Extended Model of the Human Atrioventricular Node
}

\author{
Mikael Wallman ${ }^{1}$, Frida Sandberg ${ }^{2}$ \\ ${ }^{1}$ Fraunhofer-Chalmers Centre, Gothenburg, Sweden \\ ${ }^{2}$ Department of Biomedical Engineering and Center for Integrative Electrocardiology (CIEL), Lund \\ University, Lund, Sweden
}

\begin{abstract}
The atrioventricular $(A V)$ node plays a crucial role during many supraventricular tachycardias (SVT). To better understand its function under these complex conditions, mathematical modelling has emerged as a valuable tool. The model presented here builds on a recently published $1 D$ model of the human AV-node, consisting of a series of interacting nodes, each with separate dynamics in refractory time and conduction delay. Here, we extend the formulation to $2 \mathrm{D}$ and demonstrate its ability to reproduce clinical data. Subsequently, we use it to study how $A V$ nodal properties for clinically assessed single and dual $A V$ node physiology affect activation for regular and stochastic input. In particular we study the effect of functional gradients within the AV node on ventricular response during atrial pacing and atrial fibrillation. Simulation results display important emergent features such as pathway switching and concealed conduction, and show differences in AF response that are not present in response to pacing. Simulation of a single impulse takes around $30 \mathrm{~ms}$, admitting interactive use on clinical time scales as well as parameter estimation and uncertainty quantification. To our knowledge, the presented model is the first spatially extended human AV-node model, and as such represents a novel tool for understanding the human AV-nodal function in both healthy and diseased individuals, thereby paving the way for improved SVT diagnosis and therapy.
\end{abstract}

\section{Introduction}

The atrioventricular node $(\mathrm{AVN})$ is a part of the electrical conduction system of the heart that connects the atria to the ventricles. During normal sinus rhythm, its main function is to delay electrical impulses to coordinate atrial and ventricular contractions. During supraventricular tachycardias, the refractoriness of the AV-node becomes important since it regulates which atrial impulses reach the ventricles and thus prevents the heart from racing. In spite of its prominent role in both normal and diseased cardiac function, the human AV-node is still neither routinely characterized in clinic nor well understood [1]. A deeper understanding and better characterization of the AV-nodal function will give new possibilities for improved and individualized therapy, e.g. in rate control of AF.

Currently, the AVN is thought to have at least two accessory pathways, the fast pathway (FP) and slow pathway (SP), connecting to a central node [2]. SP and FP have distinct electrophysiological properties: FP conducts impulses faster than SP, but has a longer refractory period than SP. This results in a functional interplay between the pathways, causing the complex behaviour that is observed in clinical and experimental settings. This includes concealed conduction, where impulses through one of the pathways modulate conduction of subsequent impulses without activating the ventricles, and pathway switching, where the frequency of impulses from the atria regulate which pathway is taken for AV conduction.

Although the human AVN has gained relatively little attention within the modelling community, a few previous models exist. These represent several levels of abstraction, ranging from lumped single pathway formulations [3], via statistical descriptions $[4,5]$, to highly complex biophysical models [6]. However, in all of these models, insight into the AV-nodal function is hampered either by lumping of functionally important entities, such as refractory time and conduction delay[4,5], or SP and FP conduction[3], or by computational and structural complexity[6].

To remedy this, we have recently proposed to model the human AVN function as impulse propagation in a connected graph [7]. While the model is complex enough to give non-trivial mechanistic insights into the AV-nodal function under various stimuli with minimal computational efforts, it relies on a simplified 1D geometry consisting of two pathways joining at their terminal node. Here we extend the model to a human image-derived 2D geometry, permitting us to study spatial properties of the AV-nodal activation. Using this model, estimate parameters from invasive clinical data, and study the impact of gradients of functional properties within the AVN on AV-conduction during AF. 


\section{Methods}

\subsection{Model description}

In the proposed model [7] the AV-node is described as a network of nodes $n_{j}, j=1 \ldots M$, each corresponding to a localized part of the AV-node. The nodes in the network propagate impulses, corresponding to action potentials, along the network edges. When a node receives an impulse, a new refractory period $(R)$ and delay $(D)$ are computed according to Eqs. (1) and (2).

$$
\begin{gathered}
R_{i}=R_{\text {min }}+\Delta R\left(1-e^{-\left(t_{i}-t_{i-1}-R_{i-1}\right) / \tau_{R}}\right), \\
D_{i}=D_{\min }+\Delta D e^{-\left(t_{i}-t_{i-1}-R_{i-1}\right) / \tau_{D}} .
\end{gathered}
$$

Here, $R_{i}$ and $D_{i}$ are the refractory period and delay, respectively, following impulse $i$. The parameters $R_{\text {min }}$ and $D_{\min }$ are the smallest possible refractory period and delay, and $\Delta R$ and $\Delta D$ are the maximal prolongations in refractory period and delay. The term $t_{i}$ is the arrival time of impulse $i$. Finally, $\tau_{R}$ and $\tau_{D}$ are time constants. Using Eqs. (1) and (2), impulses are propagated through the network in an event-based fashion. Impulse propagation can be efficiently evaluated using Alg. 1.

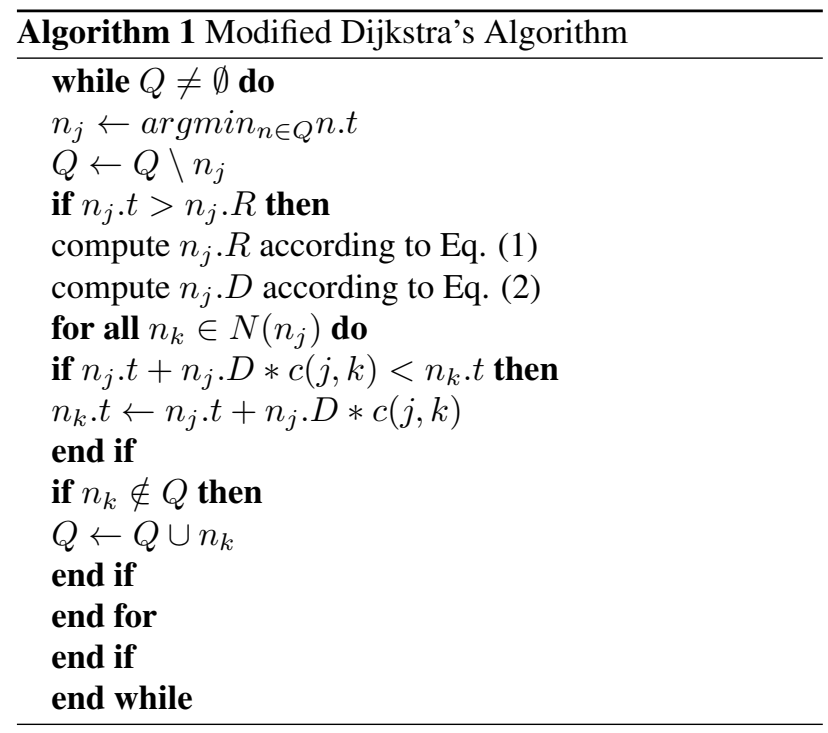

The impulse arrival time $t$, as well as $R$ and $D$, are given in point notation when associated with a specific node, so that for instance $n_{j} . t$ denotes the impulse arrival time at node $n_{j}$. Further, $N\left(n_{j}\right)$ is a function returning the neighbours of node $n_{j}$ and $c(j, k)$ is a multiplicative factor accounting for the distance between nodes $n_{j}$ and $n_{k}$. For further details, see [7].

In this work, we have used an image-derived human 2D geometrical model. The model, which was constructed from data published in [2], consists of 1044 interconnected network nodes, representing the leftward and rightward nodal extensions and the central node, measuring around $5 \mathrm{~mm}$ in both width and height. In the 2D geometry, all nodes (except the ones on the edges) are connected to 8 neighbours, with the value of $c(n, k)$ set to 1 for horizontal and vertical connections, and to $\sqrt{2}$ for diagonal connections. Two sets of parameters were used in the model, one for FP and another for SP giving a total of 12 model parameters. We denote the parameter vector $\boldsymbol{\theta}=\left[R_{\text {min }}^{F P}\right.$, $\Delta R^{F P}, \tau_{R}^{F P}, R_{m i n}^{S P}, \Delta R^{S P}, \tau_{R}^{S P}, D_{\min }^{F P}, \Delta D^{F P}, \tau_{D}^{F P}$, $\left.D_{\text {min }}^{S P}, \Delta D^{S P}, \tau_{D}^{S P}\right]$. Two parameterizations were compared: in one, parameters were interpolated between these values to create a smooth gradient, as shown in Fig. 1(a), where a value of 1 represent FP parameters, a value of 0 represents SP parameters and values in between represent linear combinations of the two. Gradients of connexin expression, thought to correlate with functional properties, have been observed in the human AV-node [2]. In the other parametrization, values were set to either 1 or 0 , creating a sharp boundary between SP and FP parameters, as shown in Fig. 1(b).
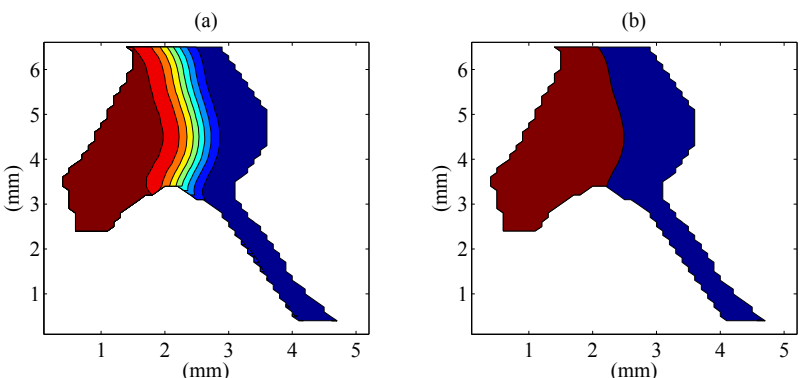

Figure 1. Geometry and distribution of functional properties of the 2D model, consisting of a leftward extension (FP) and a rightward extension (SP). Dark red and dark blue represents functional properties of FP and SP respectively. Panel (a) shows a smooth gradient of parameters between SP and SP, whereas panel (b) shows the setting with a sharp functional boundary.

\subsection{Simulations}

Initially, we fitted model parameters to two data sets, corresponding to two different patients, one suffering from AVNRT (patient 1) and one without supraventricular arrhythmias (patient 2), presented in [8]. This was done under the two different hypotheses about distribution of functional properties of the AVN, as show in in Fig. 1. To visualize the four resulting parameter sets, graphs of Eqs. 1 and 2 under a range of diastolic intervals (corresponding to $\left(t_{i}-t_{i-1}-R_{i-1}\right)$ in Eqs. 1 and 2$)$ are shown in Fig. 2. Clinical data together with corresponding model output are displayed in Fig. 3 in the next section. 

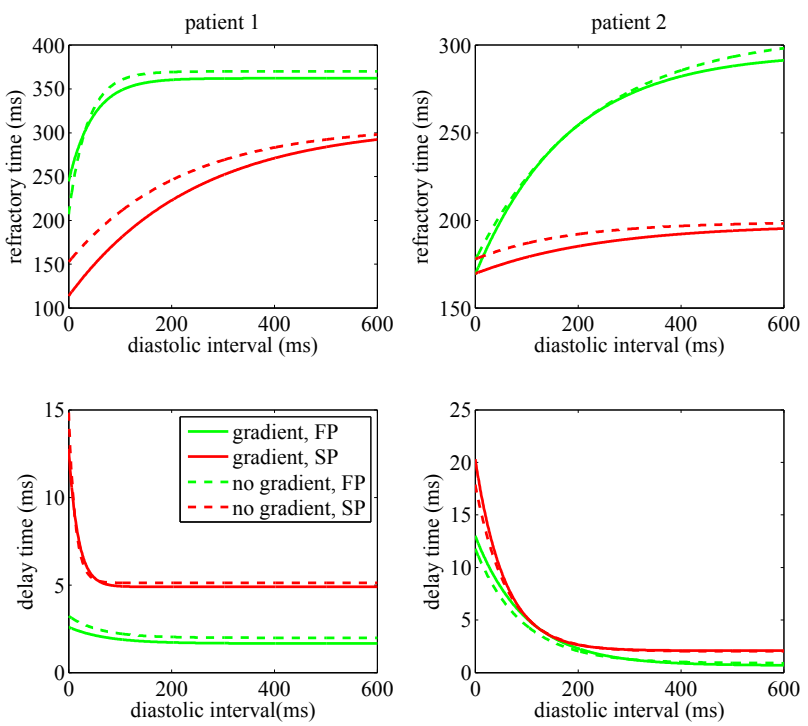

Figure 2. Refrectory and delay curves per node for SP and FP. Green lines correspond to FP, red lines correspond to SP, solid lines indicate the presence of a parameter gradient, whereas dashed lines correspond to the case with a sharp boundary between SP and FP.

Next, to investigate the behaviour of the model under arrhythmia, impulses arriving at the AVN as a result of $\mathrm{AF}$ were simulated using a Poisson process. The single parameters of the process, $\lambda$, corresponds to the average arrival frequency of impulses, and is thus intimately related to the fibrillation frequency. To account for the refractoriness of atrial tissue undergoing fibrillation, we set a lower limit of $50 \mathrm{~ms}$ on the interval between two consecutive impulses (AA-interval) [4]. Using this setup, we used the 2D model to investigate the behaviour of the four parameter sets under $\lambda$ set to $3 \mathrm{~Hz}$ and $6 \mathrm{~Hz}$.

\section{Results}

Simulation results from the S1S2 pacing scenario and corresponding clinical data are displayed in Fig. 3. As visible from the figure, the simulation results for all parameter sets replicate the clinical data almost perfectly for both patients.

The 2D model also permits investigation of the activation patterns resulting from individual beats. In Fig. 4 we show activation isochrones corresponding to the two impulses on either side of the discontinuity in the left panel of Fig. 3. Results are shown in the presence of a gradient of functional properties, but the mechanism is identical for the case with a sharp boundary.

Figure 4 elucidates the mechanism behind the pathway switching. In panel (a), both pathways are simultaneously activated. However, because of the faster conduction of FP the impulse from FP manages to penetrate some distance
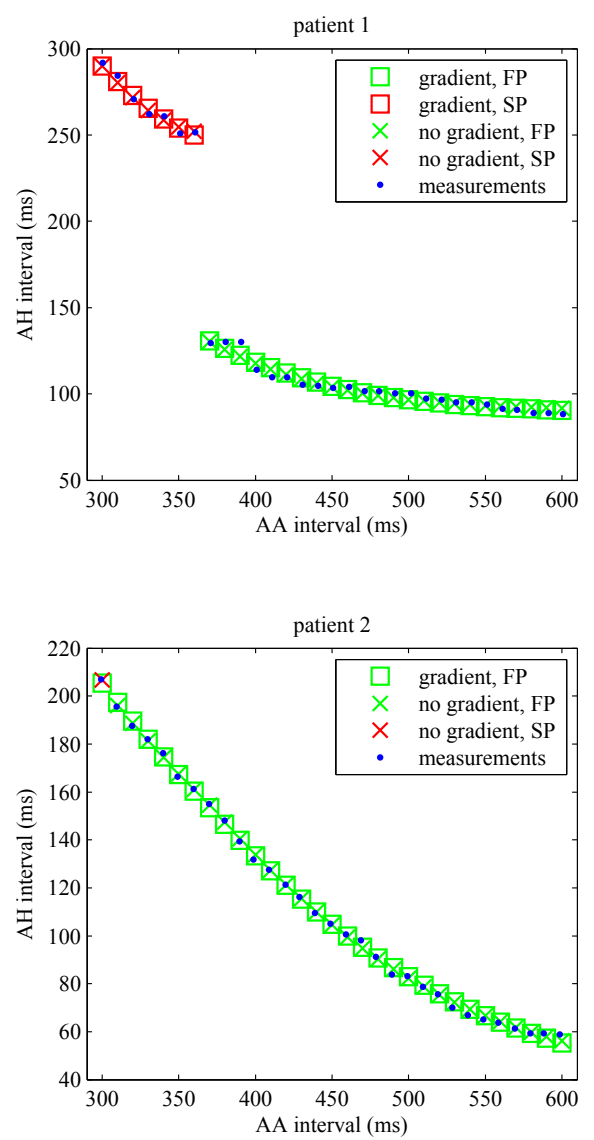

Figure 3. AH-intervals from a standard S1S2 protocol, derived from invasive measurements. Clinical data is shown as blue dots, whereas simulation results are shown as green (FP) and red (SP) x (no gradient) and squares (gradient). (a)

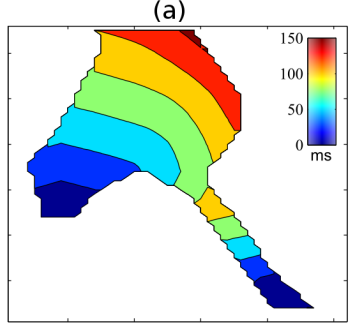

(b)

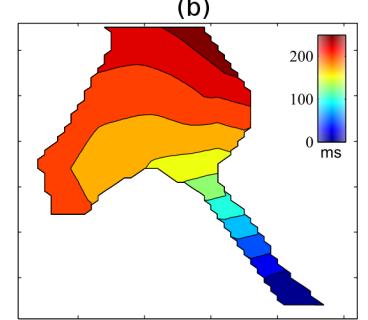

Figure 4. Panels (a) and (b) show activation isochrones during and S1S2 protocol, illustrating rate dependent switching of conduction from the leftward extension (in panel a) to the rightward extension (in panel b).

into SP before terminating through head-to-head interaction with the wavefront from SP. This also illustrates the concealed conduction phenomenon. Conversely, in panel (b), the S1S2 has reached the effective refractory period of FP, which fails to activate at all. This prevents the wavefront collision, allowing the SP impulse to propagate and resulting in a switching of the pathways. 
Next, we investigated the model behaviour under AF conditions, using a Poisson process to generate impulses as described in Sec. 2.2. For each virtual patient, we tested two fibrillation frequencies: $\lambda=3 \mathrm{~Hz}$ and $\lambda=6 \mathrm{~Hz}$. Figure 5 shows the model output as histograms of $\mathrm{HH}$ intervals.
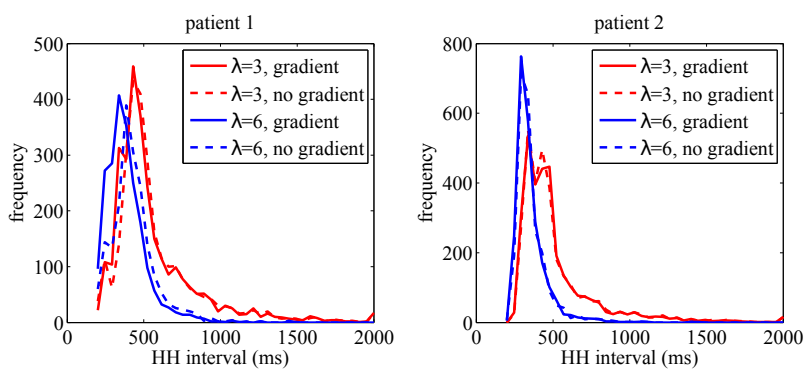

Figure 5. HH-intervals for the two virtual patients, under different Poisson processes ( $\lambda=3$, red lines, and $\lambda=6$, blue lines) and in the presence (solid lines) and absence of a functional gradient (dashed lines).

As visible in the Fig. 5, increasing $\lambda$ results in a narrowing of the histograms and a translation towards smaller $\mathrm{HH}$ intervals. An interesting difference between the patients is the sensitivity to the functional gradient. In the left panel, patient 1 displays a clear difference between the presence and absence of the gradient for $\lambda=6 \mathrm{~Hz}$.

Table 1, displaying numerical statistics for impulse propagation in the AF scenario, corroborate these results. Statistics for $\lambda=3 \mathrm{~Hz}$ are similar regardless of the presence or absence of a functional gradient. Contrastingly, for $\lambda=6$ $\mathrm{Hz}$, the presence of a gradient leads to a lowering of the average $\mathrm{HH}$-interval and an increased conduction through SP in patient 1 . Corresponding data for patient 2 also shows an increase in SP conduction, but does not display any significant effects on the average $\mathrm{HH}$-interval.

\section{Conclusions}

We have presented a 2D model of the human AV-node, and demonstrated its ability to replicate invasive clinical data. Additionally, we have investigated the behaviour of the model under AF conditions, showing its ability to produce realistic HH histograms. Finally, we have used the model to investigate the impact of the distribution of functional properties within the $\mathrm{AV}$-node. Results indicate that even though model output looks identical in a pacing study, the effect of subjecting the AV node to stochastic impulses from AF may differ. In our study, this was especially evident when pacing provoked a discontinuity in $\mathrm{AH}$ delay during an S1S2 protocol, corresponding to a clinical indication of dual AV node physiology. However, even in the absence of such a discontinuity, we demonstrated that dual pathways with different functional properties may exist and play a role in $\mathrm{AV}$ conduction during $\mathrm{AF}$.
Table 1. Statistics of AV conduction for patient 1 and 2. $\lambda$ is in $\mathrm{Hz},+$ indicates the presence of a gradient, $\mathrm{HH}$ interval average and standard deviation are given in ms. SP and FP signifies percentage of impulses conducted through the respective pathway. Block indicates percentage of nonconducted atrial impulses.

\begin{tabular}{cccccc}
\hline \hline$\lambda$ & grad & mean $\mathrm{HH} \pm$ std (ms) & block & SP & FP \\
\hline \multirow{5}{*}{$\begin{array}{c}\text { patient 1 } \\
3\end{array}+$} & $564 \pm 304$ & $42 \%$ & $33 \%$ & $67 \%$ \\
6 & + & $580 \pm 307$ & $44 \%$ & $33 \%$ & $67 \%$ \\
6 & - & $385 \pm 135$ & $57 \%$ & $64 \%$ & $36 \%$ \\
& $430 \pm 146$ & $62 \%$ & $58 \%$ & $42 \%$ \\
3 & + & patient 2 & & & \\
3 & - & $516 \pm 274$ & $37 \%$ & $30 \%$ & $70 \%$ \\
6 & + & $519 \pm 277$ & $38 \%$ & $27 \%$ & $73 \%$ \\
6 & - & $353 \pm 105$ & $54 \%$ & $56 \%$ & $44 \%$ \\
\hline \hline
\end{tabular}

\section{References}

[1] Billette J, Tadros R. Integrated rate-dependent and dual pathway AV nodal functions: principles and assessment framework. Am J Physiol Heart Circ Physiol 2014;306:H173-83.

[2] Kurian T, Ambrosi C, Hucker W, Fedorov VV, Efimov IR. Anatomy and electrophysiology of the human av node. Pacing Clin Electrophysiol 2010;33:754-762.

[3] Lian J, Müssig D, Lang V. Computer Modeling of Ventricular Rhythm During Atrial Fibrillation and Ventricular Pacing. IEEE Trans Biomed Eng 2006;53:1512-1520.

[4] Corino VDA, Sandberg F, Mainardi LT, Sörnmo L. An atrioventricular node model for analysis of the ventricular response during atrial fibrillation. IEEE Trans Biomed Eng 2011;58:3386-3395.

[5] Henriksson M, Corino V, Sörnmo L, Sandberg F. A Statistical Atrioventricular Node Model Accounting for Pathway Switching During Atrial Fibrillation. IEEE Trans Biomed Eng 2016;63:1842-1849.

[6] Inada S, Boyett M, Dobrzynski H. Mathematical models of human sinus and atrioventricular node action potentials. In Computers in Cardiology, 2009. 2009; 77-80.

[7] Wallman M, Sandberg F. Characterization of human avnodal properties using a network model. Medical biological engineering computing 2017; In press.

[8] Liu S, Olsson SB, Yang Y, Hertervig E, Kongstad O, Yuan S. Concealed conduction and dual pathway physiology of the atrioventricular node. J Cardiovasc Electrophysiol February 2004;15(2):144-149. ISSN 1045-3873.

Address for correspondence:

Mikael Wallman

Fraunhofer-Chalmers Centre

Sven Hultins gata 9, 41258 Gothenburg, SWEDEN

miwa@fcc.chalmers.se 